\title{
REMAT e a gestão editorial de 2019: da ampliação de indexadores à publicação em fluxo contínuo
}

\author{
Remat and the 2019 editorial management: from expanding indexers to the \\ publication in the "rolling pass" format
}

\author{
Glauciane Klein Burgiert Padilha \\ Graduanda em Matemática \\ Instituto Federal de Educação, Ciência e Tecnologia do Rio Grande do Sul \\ glauciane.padilha@caxias.ifrs.edu.br \\ Greice da Silva Lorenzzetti Andreis \\ Doutora em Engenharia Química \\ Instituto Federal de Educação, Ciência e Tecnologia do Rio Grande do Sul \\ greice.andreis@caxias.ifrs.edu.br \\ Katia Arcaro \\ Doutora em Matemática Aplicada \\ Instituto Federal de Educação, Ciência e Tecnologia do Rio Grande do Sul \\ katia.arcaro@caxias.ifrs.edu.br \\ Daiane Scopel Boff \\ Doutora em Educação \\ Instituto Federal de Educação, Ciência e Tecnologia do Rio Grande do Sul \\ daiane.boff@caxias.ifrs.edu.br
}

\begin{abstract}
Resumo à qualidade.

Palavras-chave

REMAT. Indexadores. OJS 3. DOI. Publicação em Fluxo Contínuo.
\end{abstract}

Este artigo tem por objetivo historicizar a trajetória da REMAT: Revista Eletrônica da Matemática com o registro das principais ações realizadas em 2019/2020, que incluem a ampliação do número de indexadores com metadados da REMAT, a migração do sistema de gestão do fluxo editorial e publicação de periódicos científicos na web, passando do Open Journal Systems (OJS) 2 para o OJS 3, o depósito de metadados dos artigos da revista no sistema Digital Object Identifier (DOI) e a publicação em fluxo contínuo (rolling pass). Com sustentação analítica, descreve-se a forma pela qual os processos de editoração são realizados e projetam-se as melhorias que podem ser obtidas por meio do upgrade do sistema OJS. O artigo apresenta também o Sistema DOI e suas vantagens, e trata da publicação em fluxo contínuo, que tem por objetivo principal acelerar o processo de comunicação das pesquisas, disponibilizando ao leitor conteúdo de maneira mais rápida e, consequentemente, favorecendo o aumento do número de citações. A partir dessas ações esperase qualificar o fluxo editorial para autores, avaliadores e editores, e ampliar a divulgação das pesquisas entre os leitores, fazendo com que a REMAT continue em ascensão no que tange à visibilidade e, principalmente,

doi: $10.28998 /$ cirev.2020.7ne.37-46

Esta obra está licenciada sob uma Licença Creative Commons.

Submetido em: 09/03/2020

Aceito em: $14 / 03 / 2020$

Publicado em: 30/03/2020 


\begin{abstract}
This article aims to historicize the trajectory of REMAT: Revista Eletrônica da Matematica with the record of the main actions carried out in 2019/2020, which include the expansion of the number of indexers with REMAT metadata, the migration of the publication and editorial flow management system of scientific journals on the web, changing the Open Journal Systems (OJS) 2 to OJS 3, the deposit of metadata for the journal's articles in the Digital Object Identifier (DOI) system and the publication in a "rolling pass" format. With analytical support, it is described the way in which the publishing processes are carried out and the improvements that can be obtained by upgrading the OJS system are designed. The DOI System and its advantages are also presented, and it is addressed the issue of the publication in a "rolling pass" format, whose main objective is to accelerate the research communication process, making content available to the reader more quickly and consequently, favoring an increase in the number of citations. Based on these actions, it is expected to qualify the editorial flow for authors, reviewers and editors, and to expand the dissemination of research among readers, making REMAT to continue to rise in terms of visibility and, mainly, quality.
\end{abstract}

\title{
Keywords
}

REMAT. Indexers. OJS 3. DOI. Rolling Pass.

\section{INTRODUÇÃO}

A REMAT (e-ISSN 2447-2689, DOI 10.35819) teve início em 2015, e tem o objetivo de publicizar produções originais em três seções: Matemática em Contextos Técnicos e/ou Tecnológicos, Ensino de Matemática e Matemática Pura e/ou Aplicada. Como meio de divulgação científica de um periódico exclusivamente eletrônico, a REMAT enquadra-se no que Lancaster (1995) define como "jornal eletrônico" e, como tal, pode oferecer alguns recursos a mais do que os meios impressos oferecem, como mecanismos de busca, links entre fontes eletrônicas e até mesmo a fácil manipulação de dados. Dentre as vantagens dos jornais eletrônicos, Lancaster (1995) destaca o menor custo de manutenção, publicações mais rápidas de resultados de pesquisa e fácil comunicação entre pesquisadores e leitores. Nesse sentido, a REMAT vem investindo na missão de compartilhar com qualidade e eficiência produções científicas que envolvem práticas educativas e resultados de pesquisas relacionadas à Matemática (VISSIRINI et al., 2019). Garantindo acesso livre às suas publicações até então semestrais, a Equipe Editorial entende a importância de difundir os achados científicos e as experiências educativas de sucesso não só na esfera acadêmica, mas usufruir da vantagem do meio eletrônico para disponibilizar à comunidade em geral o que vem sendo pensado e desenvolvido na academia. Nesse sentido, a partir de janeiro de 2020 a REMAT mudou sua periodicidade de publicação de semestral para a publicação em fluxo contínuo.

Além da facilidade de acesso e disseminação que o jornal eletrônico proporciona, Lancaster (1995) já entrevia, há quase 25 anos, que esse meio viria a oportunizar maneiras inovadoras de apresentar resultados de pesquisas, como o uso de animações, sons, links entre fontes, entre outros. Embora não citadas diretamente pelo autor, entre as possibilidades que o meio impresso comum não dispõe, estão as ferramentas de acessibilidade. Nesse contexto, e com vistas a ampliar o alcance da divulgação científica, a REMAT também vem investindo na acessibilidade digital (SILVA et al., 2018).

Outra vantagem do jornal eletrônico, segundo Lancaster (1995), é a facilidade no processo de avaliação por pares e de comunicação entre autores, editores e avaliadores. A questão do processo de avaliação por pares e os problemas intrínsecos a ele são bastante discutidos já há algum tempo (COSTA, 1996; HARNAD, 1996; CAMPANARIO, 2002); no entanto, é a metodologia de avaliação de produções científicas que perdura, sendo a mais utilizada atualmente (PATRUS; DANTAS; SHIGAKI, 2016) e, mais do que isso, “[...] já há algum consenso de que o julgamento pelos pares "[...] pode ser implementado nos meios eletrônicos, emprestando ao periódico eletrônico o mesmo reconhecimento do periódico impresso" (COSTA, 1996, p. 5). Auxiliando na dinâmica desse processo que, mesmo facilitado, segue sendo complexo e intrincado na medida em que há uma real preocupação com a ética, a imparcialidade e a qualidade do material a ser publicado, são criados os sistemas de gerenciamento e publicação de revistas eletrônicas. Entre os de acesso 
livre destaca-se o Open Journal Systems (OJS) que, só no Brasil, conta com mais de 1700 revistas usuárias da ferramenta (BRITO et al., 2018).

Desde a sua criação até então, a REMAT vinha fazendo uso do OJS 2, que intermediava integralmente todo o processo de submissão, comunicação entre autores e editores, e entre esses últimos com avaliadores, até a edição final e, então, a publicação. Recentemente a instituição fomentadora ${ }^{1}$ da REMAT optou por migrar para a versão 3 do OJS e realizar a atribuição do Digital Object Identifier (DOI) para as publicações do Portal de Periódicos do IFRS, no qual a REMAT inclui-se. Além disso, visando uma maior abrangência, no último ano os metadados dos artigos científicos publicados pela REMAT foram disponibilizados em mais duas bases de dados, o Portal de Periódicos CAPES e o Latindex.

Todas essas ações fizeram com que o número de acessos aos artigos publicados crescesse consideravelmente a partir do segundo semestre de 2019. Na sequência deste texto, descrevem-se as perspectivas de melhoria da revista e as novas possibilidades de avanço frente às inovações adotadas.

\section{REMAT: indexação e maior visibilidade}

A REMAT é um periódico indexado em bases de dados e diretórios, nas quais seu conteúdo temático é descrito. São fornecidas informações dos artigos, tais como: autor e título do artigo, título e local de publicação do periódico, volume, número e as páginas onde se encontra o artigo. Essas bases permitem a recuperação e a ampliação da visibilidade dos títulos indexados, assim como atribuem ao periódico um valor técnico, tendo em vista que as informações publicadas na REMAT passam por uma avaliação criteriosa, dando ao periódico maior credibilidade.

Santos (2011) apresenta um guia sobre fontes de indexação para periódicos científicos indicando 85 bases de dados das mais diversas áreas do conhecimento e 11 diretórios de áreas multidisciplinares e especializadas de fontes nacionais e estrangeiras. Segundo o autor,

A base de dados, especificamente, é a seleção de parte ou do total de outra coleção de dados, constituída, no mínimo, por um arquivo e concebida para determinado fim ou para um dado sistema de processamento de dados. [...] Os diretórios são listas que registram novos títulos; mudanças de título; títulos a serem lançados; fusões de valores descritivos; e outras características relevantes para os periódicos científicos, instrumentos de divulgação técnico-profissional e científico e cultural que são publicados em vários países (SANTOS, 2011, p. 6).

A seguir, apresentam-se as bases de dados e os diretórios em que a REMAT está indexada, por ordem de cadastro. A maioria delas encontra-se no levantamento realizado por Santos (2011):

- Google Acadêmico;

- Diretório de Políticas Editoriais das Revistas Científicas Brasileiras - Diadorim;

- Instituto Brasileiro de Informação em Ciência e Tecnologia - ibict oasisbr;

- Revistas de livre acesso - Livre;

- Sumários de Revistas Brasileiras - sumários.org;

- Directory of open access journals - DOAJ;

- Portal de Periódicos CAPES;

- Diretório do Sistema Latindex: Sistema Regional de Información en Línea para Revistas Científicas de América Latina, el Caribe, España y Portugal.

Além da visibilidade oportunizada pela indexação, o Sistema DOI é um atrativo a autores e leitores pelas vantagens que oferece. Conforme Brito et al. (2015, p. 9),

\footnotetext{
${ }^{1}$ Instituto Federal de Educação, Ciência e Tecnologia do Rio Grande do Sul (IFRS).
} 
Trata-se de um identificador persistente, único e publicado que gestores de conteúdo vinculam a objetos físicos ou digitais, o que possibilita ofertar serviços e garantir a propriedade intelectual, principalmente para objetos digitais disponíveis na Internet. [...] O uso de um identificador para entidades digitais, como artigos científicos ou conjuntos de dados, permite a implementação de serviços associados aos metadados depositados junto ao identificador. Serviços como localização, gestão de direitos autorais, métricas de acesso e de referenciamento são úteis e garantem acesso ao texto integral, da mesma forma em que se preservam os metadados em base de dados, ofertando-se certa segurança em caso de contingência e alinhando-se com as necessidades de curadoria de conteúdos digitais na Internet.

Existem diferentes agências de registro DOI que gerenciam esses identificadores e ofertam serviços, de modo que "[...] as agências de registro que fazem parte do sistema DOI são responsáveis pela inserção e armazenamento dos metadados depositados" (BRITO et al., 2015, p. 12). Os registros DOI da REMAT são gerenciados pela Crossref por intermédio da Associação Brasileira de Editores Científicos (ABEC). A Crossrefé uma organização que

[...] restringe sua operação à identificação e manutenção de informações (metadados) de documentação científica, com uma base de dados que possibilita ligar referências digitais. Não mantém textos completos, apenas as referências, com destaque para a questão de autoria e localização. Assim, assegura confiabilidade na citação de documentos disponibilizados na Internet (BRITO et al., 2015, p. 13).

A atribuição do DOI foi realizada para todas as publicações da revista, desde o volume 1, número 1, de 2015. Na configuração do endereço eletrônico, incluíram-se o nome da revista, o ano de publicação, o volume, o número da edição e o número de identificação do artigo na revista. Como exemplo, o endereço eletrônico de um dos primeiros artigos publicados no formato de publicação contínua foi: https://doi.org/10.35819/remat2020v6ilid3524.

De acordo com o Relatório de Acessos da REMAT gerado pelo OJS, foram contabilizados 86.700 acessos aos arquivos dos artigos de 2015 a 31 de dezembro de 2019. No Gráfico 1 pode-se observar a evolução no número de acessos por ano. 
Gráfico 1 - Número de acessos anuais aos artigos da REMAT.

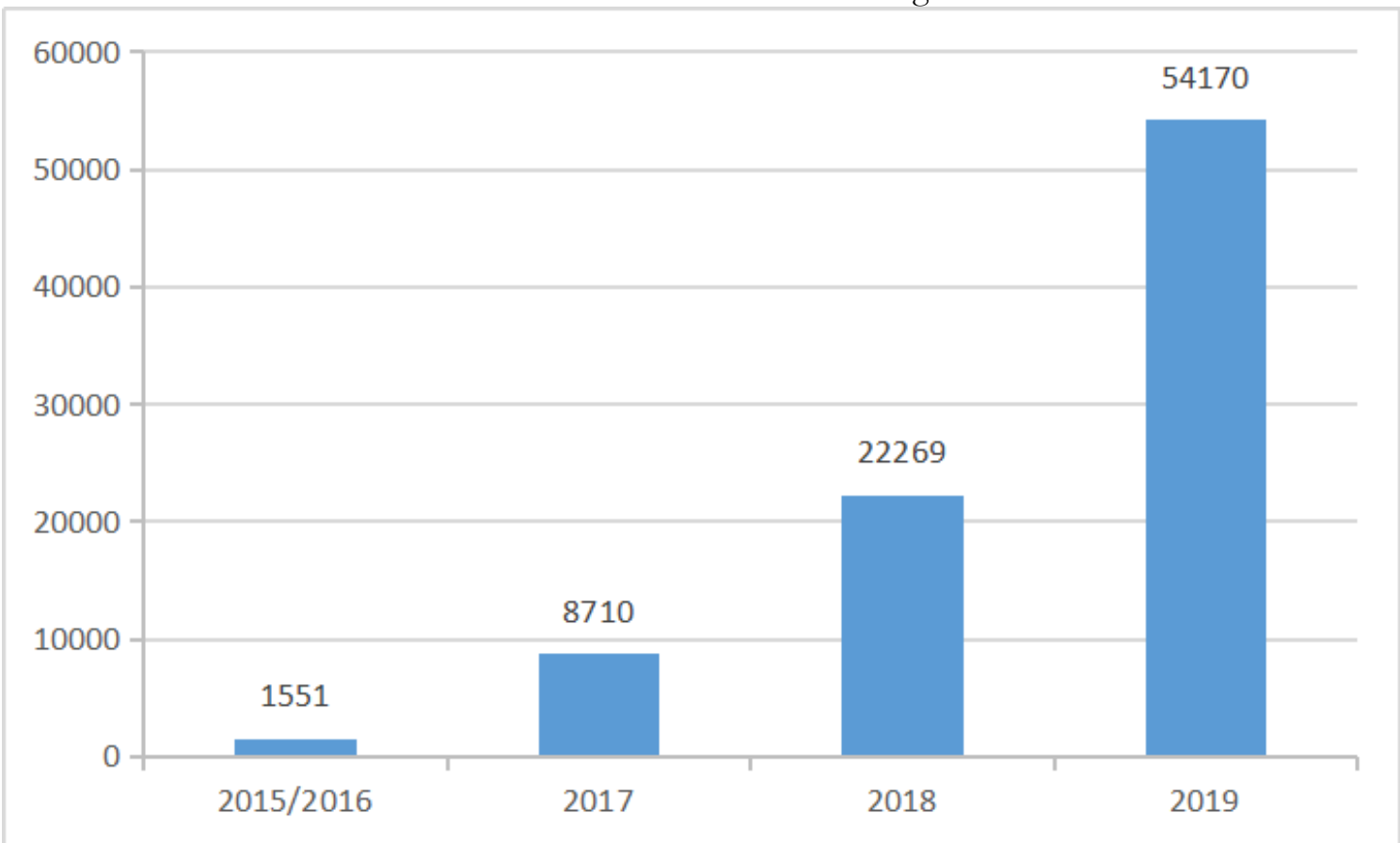

Fonte: Dados do Relatório de Acessos da REMAT de 2015 a 31 de dezembro de 2019 (2019).

Em 2019, a REMAT foi incluída no Portal de Periódicos da Capes, no Latindex, e os metadados de seus artigos foram depositados no Sistema DOI, o que impulsionou o número de acessos no último semestre, como mostra o Gráfico 2.

Gráfico 2 - Número de acessos mensais aos artigos da REMAT em 2019.

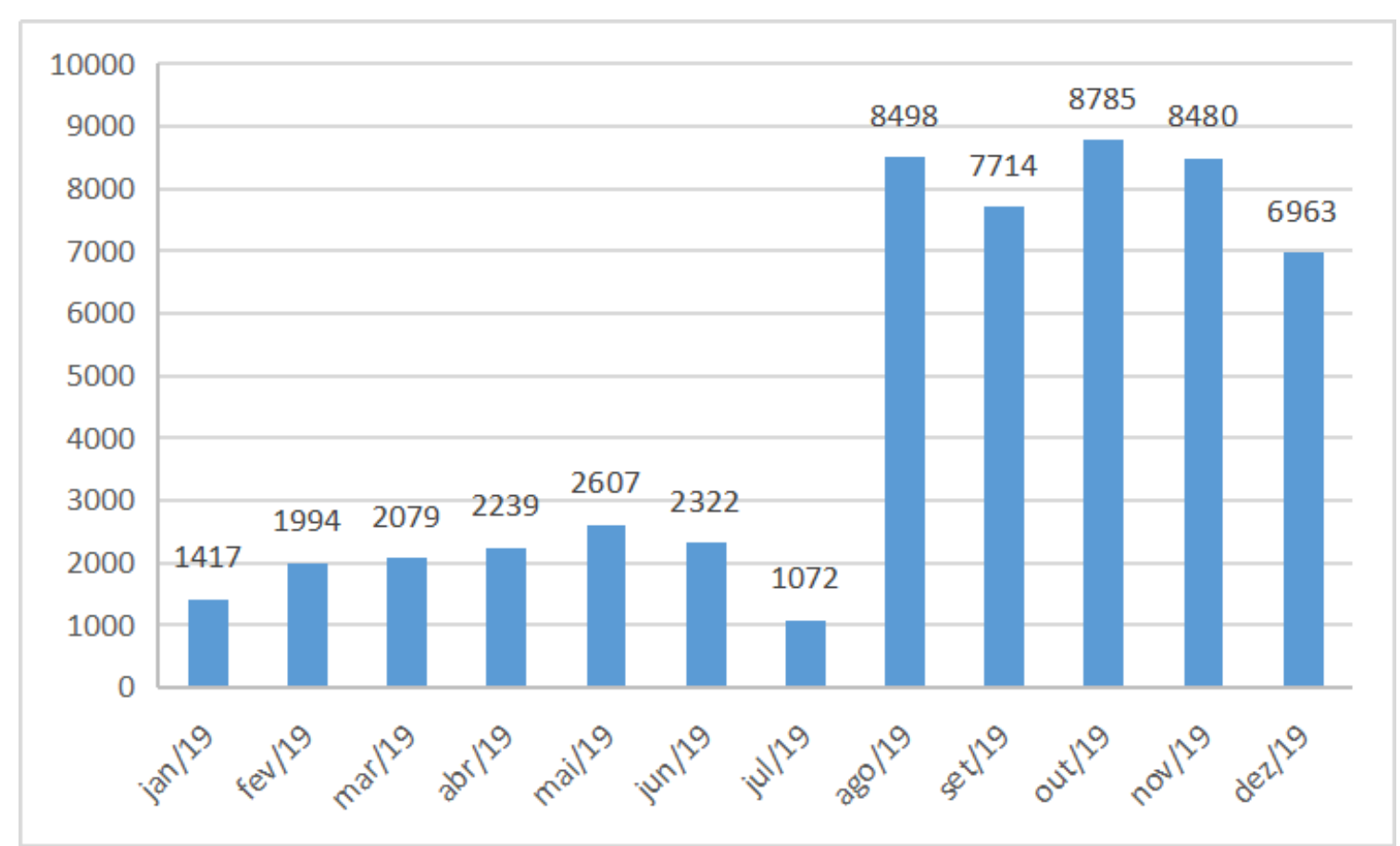

Fonte: Dados do Relatório de Acessos da REMAT (2019).

A partir da visibilidade obtida, entende-se que o cadastro em outras bases de dados e diretórios continua sendo uma meta para a REMAT, além da manutenção do Sistema DOI. 


\section{MIGRAÇÃO PARA O OJS 3}

A REMAT utiliza o software de código livre OJS para gerenciamento e publicação da revista eletrônica. Para fazer melhorias no processo editorial e aperfeiçoar a experiência de uso e acesso dos usuários, o Portal de Periódicos do IFRS realizou o upgrade do sistema com a migração da versão OJS 2 para a versão OJS 3 (versão 3.1.1.4) em julho de 2019. Entre as principais novidades com maior impacto na REMAT, destacam-se: inscrição do ORCID dos autores no cadastro de usuário do portal; adoção do framework Bootstrap; flexibilização do processo de editoração; possibilidade de discussões entre perfis e a viabilização para os usuários exercerem diversas funções dentro da revista sem a necessidade de criar outros cadastros ou alternar entre papéis no portal (BRITO et al., 2018). A descrição de cada uma dessas melhorias apresenta-se na Tabela 1. Essas informações foram compiladas de Brito et al. (2018).

Tabela 1 - Atualizações no OJS 3.

\begin{tabular}{|l|l|}
\hline \multicolumn{1}{|c|}{ Melhoria } & \multicolumn{1}{c|}{ Descrição } \\
\hline Framework Bootstrap & $\begin{array}{l}\text { Permite que páginas HTML adaptem-se a dispositivos móveis. } \\
\text { Assim, em qualquer dispositivo tem-se um visual com todas as } \\
\text { funcionalidades por meio de temas responsivos que podem ser } \\
\text { personalizados. A técnica possibilita que o OJS 3 seja responsivo aos } \\
\text { atuais dispositivos. }\end{array}$ \\
\hline Editoração & $\begin{array}{l}\text { A atualização do sistema permite a flexibilização do processo de } \\
\text { editoração. É possível transitar entre as etapas no processo e } \\
\text { personalizar o fluxo editorial. }\end{array}$ \\
\hline Discussões & $\begin{array}{l}\text { Visando aperfeiçoar o controle das comunicações, o OJS 3 tem a } \\
\text { possibilidade de criar uma discussão interna para cada uma das fases } \\
\text { do fluxo editorial: submissão, revisão, edição de texto e produção. } \\
\text { Essa ferramenta permite que um avaliador crie um tópico de } \\
\text { discussão convidando um editor de seção, por exemplo, para } \\
\text { participar. }\end{array}$ \\
\hline Cadastro de usuários & $\begin{array}{l}\text { O cadastro de usuários em OJS 3 é interoperável com o ORCID, } \\
\text { que é o identificador digital do autor. Desta forma, ao se cadastrar } \\
\text { no OJS 3 basta o usuário se conectar no ORCID que os demais } \\
\text { campos do formulário de cadastro serão automaticamente } \\
\text { preenchidos. }\end{array}$ \\
\hline Funções & $\begin{array}{l}\text { Usuários podem executar atividades pertencentes a mais de um } \\
\text { papel. O OJS 3 permite ao Gerente da Revista criar novos papéis, } \\
\text { ou mesmo renomear os existentes, sendo mais fácil gerenciar os } \\
\text { usuários do sistema dando as permissões necessárias a cada papel de } \\
\text { usuário. Outra melhoria associada é o usuário não precisar mudar de } \\
\text { papel para realizar diferentes tarefas. }\end{array}$ \\
\hline
\end{tabular}

Fonte: Adaptação de Brito et al. (2018).

Para garantir a fluidez do processo migratório, foi estruturado um plano estratégico 5W2H focando as etapas e ações necessárias para concretizar a migração do sistema OJS 2 para o OJS 3. Partindo da pesquisa inicial acerca do desempenho do software e implicações da atualização, definiram-se quais procedimentos seriam adotados para realizar a migração, as etapas e atividades relativas a eles, seus objetivos específicos e os agentes envolvidos em cada ação. Durante o processo 
migratório foi realizada a transferência do conteúdo hospedado na versão antiga da plataforma para a atual, a manutenção dos metadados dos artigos publicados e dos usuários da revista, a avaliação do website do periódico com correções no design gráfico, a atualização das informações publicadas e a revisão das marcas estruturais de acessibilidade digital. A migração representou um período de grande desenvolvimento para a REMAT e para os envolvidos, assim como oportunizou um aprendizado para a equipe.

Fazendo uma análise geral do OJS 3 e dos resultados da atualização no Portal de Periódicos do IFRS, percebeu-se, após um período de adaptação ao novo sistema, a relativa simplicidade de seu uso, o que vai ao encontro das expectativas da Equipe Editorial no que tange à agilidade e à qualificação do trabalho editorial. De fato, certas implementações facilitaram o processo de submissão e editoração de artigos, bem como o processo de avaliação, seja para autores, editores ou avaliadores. O design do software harmoniza estrategicamente elementos dos conceitos clássico e moderno de periódicos, combinando funcionalidade e inovação tecnológica. O painel único com menu lateral auxiliou a agilizar a atividade editorial; os usuários conseguem visualizar e executar diferentes tarefas dentro do fluxo editorial sem a necessidade de trocar perfis. As discussões durante o processo de revisão dos artigos, centralizadas no fórum integrado a cada etapa editorial, simplificaram e concentraram a comunicação entre usuários. O cadastro de usuários por meio do registro ORCID trouxe como contribuição a otimização na inserção de metadados nos artigos.

Projeta-se a possibilidade de se operar o sistema pelo celular em função da adoção do framework Bootstrap no OJS 3, que torna o conteúdo publicado adequado aos recursos tecnológicos existentes. Dentre as atividades planejadas para a avaliação do processo de migração do sistema, inclui-se o monitoramento das implementações realizadas e a manutenção das versões posteriormente lançadas. Almeja-se sistematizar os dados referentes ao número de acessos aos artigos da REMAT após a atualização do OJS, além de observar em quais tipos de dispositivos foram feitos os acessos e em que localidades. A análise desses dados será utilizada posteriormente para a manutenção e a qualificação do periódico.

\section{FLUXO CONTÍNUO DE PUBLICAÇÃO}

Desde a sua criação em 2015, a REMAT vem publicando dois números por volume; o primeiro, publicado no primeiro semestre de cada ano, e o segundo, no semestre seguinte. Inicialmente, a revista operou com um período determinado para submissões. Atenta às possibilidades de melhoria no fluxo de editoração, a equipe editorial passou a aceitar submissões em fluxo contínuo, embora ainda mantivesse as publicações semestrais. Após um período de amadurecimento e planejamento, outra mudança significativa implementada foi a publicação em fluxo contínuo (rolling pass). A partir do volume 6, número 1, referente a 2020, a REMAT passa a publicar cada artigo após seu aceite, findados seus trâmites de edição de texto e leiaute. $\mathrm{O}$ fechamento de cada número será realizado ao final de cada semestre. Entende-se que a publicação em fluxo contínuo seja uma tendência que vem sendo fomentada por indexadores internacionais, uma vez que é seguida por alguns dos mais renomados jornais eletrônicos nacionais, das mais diversas áreas do conhecimento. Góis Junior et al. (2018, p. 409) explicitam que:

"Educação e Pesquisa", os “Anais do Museu Paulista", os "Arquivos do Instituto Biológico", todas publicações da Usp, [...] a "Fisioterapia em Movimento" e o "Journal of Physical Education", ambas publicações indexadas no Scielo, também adotaram o referido modelo. O próprio Portal de Periódicos Eletrônicos Científicos (PPEC) da Unicamp tem sido condutor e incentivador destas novas práticas editoriais.

Ampliando a gama de periódicos de publicação em fluxo contínuo, muitas revistas nacionais da área da Matemática e da Educação Matemática têm esse meio para divulgação de seu 
conteúdo: Zetetiké, Revista Eletrônica de Educação Matemática, Revista de Educação Matemática, NEXUS Mathematicæ, Revista de Matemática da Universidade Federal de Ouro Preto (UFOP), entre outras. A principal defesa dessa prática é a agilidade e o dinamismo da publicação dos artigos já aceitos, sem a necessidade de esperar a composição completa das edições (MIRANDA, 2017, GÓIS JUNIOR et al. 2018, PRIETO; PIETRI, 2018, SILVA; PRESSER, 2019): "O objetivo principal da publicação contínua de artigos é acelerar o processo de comunicação das pesquisas e assim contribuir para a sua disponibilidade para leitura e citação" (MIRANDA, 2017, on-line).

Silva e Presser (2019, p. 6) defendem que "não faz mais sentido as publicações eletrônicas aguardarem um período para lançar todos os artigos aprovadas em um único momento". De fato, uma vez aceito o artigo pela revista, quanto antes ele for publicado, maior "a visibilidade do periódico e, assim, sua recepção pelos potenciais leitores" (PRIETO; PIETRI, 2018, on-line) da redução do tempo entre a aprovação de um artigo e sua publicação, Silva e Presser (2019, p. 6) ainda citam como vantagens dessa prática a possibilidade de uma "maior quantidade de publicações, sem perder a qualidade" e a "diluição no tempo do volume de trabalho da equipe que trabalha após a aprovação dos artigos: revisores de idiomas, de normalização, diagramador e responsáveis pela divulgação do artigo [...]".

Nesse sentido, a expectativa em relação à publicação em fluxo contínuo é a de disponibilizar mais rapidamente à comunidade o conteúdo da revista, atendendo com mais agilidade às demandas dos autores de publicar seus resultados de pesquisa com a mesma qualidade, segurança e ética até então empregadas na revista. Para tanto, a REMAT conta com as ferramentas, especialmente de comunicação entre editores e autores do novo sistema a que está aderindo, o OJS 3.

\section{CONSIDERAÇÕES FINAIS}

Desde sua criação, a REMAT vem construindo espaço junto à comunidade acadêmica e colhendo bons resultados também em função de estar atenta às discussões realizadas pelos editores da comunidade científica quanto a inovações e adequações em seus processos editoriais. O ano de 2019 foi marcado por um crescente número de acessos e citações, potencializado pela inclusão da REMAT em indexadores e buscadores mais utilizados pela comunidade acadêmica. A migração do OJS 2 para o OJS 3, realizada em julho de 2019, traz um período de adaptação à nova interface do sistema, bem como um novo leque de possibilidades, que melhoram a interação entre autores, avaliadores e editores. Além disso, a atribuição do DOI aos artigos da REMAT em agosto de 2019 permitiu a identificação, a localização e a descrição única dos artigos, garantindo a propriedade intelectual aos autores. Nesse contexto de avanço e adaptação às possibilidades de melhoria, a REMAT ainda aposta em 2020 na publicação em fluxo contínuo, na busca de disponibilizar mais rapidamente o conteúdo dos artigos já aceitos e, ao mesmo tempo, em atender e respeitar a urgência dos autores em publicar os resultados das suas pesquisas.

\section{AGRADECIMENTOS}

Os autores agradecem o apoio financeiro concedido pela Pró-Reitoria de Pesquisa, Pósgraduação e Inovação (PROPPI) do IFRS por meio do Edital 01/2019/PROPPI - Apoio à Edição de Periódicos Científicos do IFRS.

\section{REFERÊNCIAS}

BRITO, Ronnie Fagundes et al. Guia do usuário do OJS 3. Brasília: Ibict, 2018. DOI: http://dx.doi.org/10.21452/978-85-7013-144-7. 
BRITO, R. F. de et al. Guia do Usuário do Digital Object Identifier. Brasília: Ibict, 2015. DOI: http://dx.doi.org/10.21452/978-85-7013-112-6I.

CAMPANARIO, J. M. El sistema de revisión por expertos (peer review): muchos problemas y pocas soluciones. Revista española de documentación científica, v. 25, n. 3, p. 267-285, 2002. Disponível em: http://redc.revistas.csic.es/index.php/redc/article/viewFile/107/171. Acesso em: 05 mar. 2020.

COSTA, S. M. de S. Controle de qualidade em periódicos científicos eletrônicos disponibilizados na Internet: a questão do julgamento pelos pares. Revista de Biblioteconomia de Brasília, v. 20, n. 2, p. 227-236, jul./dez. 1996. Disponível em:

https://www.brapci.inf.br/ repositorio/2010/03/pdf 41b411c77c 0008825.pdf. Acesso em: 05 mar. 2020.

GÓIS JUNIOR, E. et al. A tendência da publicação contínua nos periódicos. Conexões:

Educação Física, Esporte e Saúde, Campinas, SP, v. 16, n. 4, p. 409-410, out./dez. 2018. DOI: https://doi.org/10.20396/conex.v16i4.8654163.

HARNAD, St. Implementing Peer Review on the Net: Scientific Quality Control in Scholarly Electronic Journals. In: PEEK, R.; NEWBY, G. (Eds.). Scholarly Publication: The Electronic Frontier. Cambridge MA: MIT Press, 1996. Disponível em:

http://cogprints.org/1692/1/harnad96.peer.review.html. Acesso em: 26. jun. 2019.

LANCASTER, F. W. The evolution of electronic publishing. Library Trends, v. 43, n. 4, p. 51827, 1995. Disponível em:

https://pdfs.semanticscholar.org/3abd/9d8450b42be0870d27cf3d93cb7339b5dc02.pdf. Acesso em: 05 mar. 2020.

MIRANDA, Paulo Emílio V. de. Continuous Publication. Matéria (Rio de Janeiro), [s.l.], v. 22, n. 1, 26 out. 2017. FapUNIFESP (SciELO). http://dx.doi.org/10.1590/s1517-

707620170001.0241. DOI: http://dx.doi.org/10.1590/s1517-707620170001.0241.

PATRUS, R.; DANTAS, D. C.; SHIGAKI, H. B. Pesquisar é preciso. Publicar não é preciso: história e controvérsias sobre a avaliação por pares. Avaliação: revista da avaliação da educação superior, Campinas, v. 21, n. 3, p. 799-820, ago./nov. 2016. DOI:

http://dx.doi.org/10.1590/S1414-40772016000300008.

PRIETO, R. G.; PIETRI, É. de. Desafios e possibilidades da publicação contínua para o trabalho dos editores de periódicos científicos. Educação e Pesquisa, São Paulo, v. 44, nov. 2018. DOI: http://dx.doi.org/10.1590/s1517-970220184401001.

SANTOS, G. C. Fontes de indexação para periódicos científicos: um guia para bibliotecários e editores. Campinas: E-Color, 2011. Disponível em:

http://eprints.rclis.org/16898/1/fontesISBN9788563058188.pdf. Acesso em: 05 mar. 2020.

SILVA, L. H. R. da et al. Ações desenvolvidas na REMAT: Revista Eletrônica da Matemática com vistas à acessibilidade digital. Ciência da Informação em Revista, Maceió, v. 5, n. especial, p. 77-85, fev. 2018. DOI: http://dx.doi.org/10.21452/23580763.2018.5ne.77-85. 
SILVA, E. L. da; PRESSER, N. H. Fluxo contínuo e publicação contínua: desafios da editoração científica on-line. NAVUS Revista de Gestão e Tecnologia, Florianópolis, v. 9, n. 3, p. 5-6, jul./set. 2019. DOI: https://doi.org/10.22279/navus.2019.v9n3.p05-06.1011.

VISSIRINI, C. C. et al. REMAT: Análise do Perfil de seus Usuários por meio da Ferramenta Google Analytics e Difusão do Conhecimento Científico entre Professores e Estudantes de Matemática. Ciência da Informação em Revista, Maceió, v. 6, n. especial, p. 48-61, fev. 2019. DOI: http://dx.doi.org/10.21452/23580763.2019.6ne.48-61. 